\title{
STOKES AND NAVIER-STOKES PROBLEMS WITH NAVIER-TYPE BOUNDARY CONDITION IN $L^{P}$-SPACES
}

\author{
Hind Al BabA And ChÉRIF Amrouche
}

\begin{abstract}
Using the semigroup theory for the Stokes equation with Navier type boundary conditions developed in $[2,3]$, we first prove the maximal $L^{p}-L^{q}$ regularity for the strong, weak and very weak solutions of the inhomogeneous Stokes problem with Navier-type boundary conditions in a bounded domain $\Omega$, not necessarily simply connected. We also prove the existence of a unique local in time classical solution to the Navier Stokes problem with Navier-type boundary conditions and show that it is global in time for small initial data.
\end{abstract} 76 N10.

Mathematics subject classification (2010): 35B65, 35D30, 35D35, 35K20, 35Q30, 76D05, 76D07,

Keywords and phrases: Stokes and Navier-Stokes Problem, Navier-type boundary conditions.

\section{REFERENCES}

[1] Y. Amirat, D. BResch, J. Lemoine, J. Simon, Effect of rugosity on a flow governed by stationary Navier-Stokes equations, Quart. Appl. Math. 59, no. 4, 769-785 (2001).

[2] C. Amrouche, H. Al BabA, M. Escobedo, Analyticity of the semi-group generated by the Stokes operator with Navier-type boundary conditions on $L^{p}$-spaces in: V. D. Rădulescu, A. Sequeira \& V. A. Solonnikov (Eds.), Recent Advances in Partial Differential Equations and Applications, Contemporary Mathematics, Amer. Math. Soc., Vol. 666, Providence, RI, 2016, 23-40. doi:10.1090/conm/666/13337.

[3] C. Amrouche, H. Al BABA, M. Escobedo, Semi-group theory for the Stokes operator with Navier-type boundary conditions on $L^{p}$-spaces, Arch Rational Mech Anal, (2017) 223:881.doi:10.1007/s00205-016-1048-1.

[4] C. Amrouche, H. Al Baba, N. Seloula, Instationary Stokes problem with pressure boundary condition in $L^{p}$ - spaces, J. Evol. Equ. (2016) doi:10.1007/s00028-016-0331-9.

[5] C. Amrouche, M. Moussaoui, H. H. Nguyen, Very weak solutions for the Laplace equation, Work in progress.

[6] C. Amrouche, N. Seloula, On the Stokes equations with the Navier-type boundary conditions, Differ. Equ. Appl. 3 (2011) 581-607.

[7] H. AL BABA, Théorie des semi-groupes pour les équations de Stokes et de Navier Stokes avec des conditions aux limites de type Navier, PhD Thesis (2015) (electronic version at: http://www.theses.fr/2015PAUU3008).

[8] C. Amrouche, N. Seloula, $L^{p}$-theory for vector potentials and Sobolev's inequalities for vector fields: application to the Stokes equations with pressure boundary conditions, Math. Models Methods Appl., Sci. 23 (2013) 37-92.

[9] H. Bellout, J. Neustupa, P. Penel, On viscosity-continuous solutions of the Euler and NavierStokes equations with a Navier-type boundary condition, C. R. Math. Acad. Sci. Paris, 347 (2009), 1141-1146.

[10] W. Borchers And T. Miyakawa, $L^{2}$ decay for the Navier-Stokes flow in halfspaces, Math. Ann. 282 (1988), 139-155.

[11] D. BuCUR, E. FeIREISL. Š.NeČASOvÁ, Boundary behavior of viscous fluids: Influence of wall roughness and friction-driven boundary conditions, Arch. Rational Mech. Anal., 197, no. 1, 117-138, (2010). 
[12] D. Bucur, E. FeIreisl, Š, NeČAsová, J. Wolf, On the asymptotic limit of the Navier-Stokes system on domains with rough boundaries, J. Differential Equations, 244, no. 11, 2890-2908 (2008).

[13] T. Coulhon, D. Lamberton, Régularité $L_{p}$ pour les équations d'évolution, Séminaire d'Analyse Fonctionnelle Paris VI-VII (1984-85), 155-165.

[14] G. Dore, A. Venni, On the closedness of the sum of two closed operators, Math. Z. 196 (1987) 189-201.

[15] H. Fujita, T. Kato, On the Navier-Stokes initial value problem. I, Arch. Rational Mech. Anal., 16, $269-315,1964$.

[16] M. Geissert, H. Heck, C. TRUnK, $H^{\infty}$-calculus for a system of Laplace operators with mixed order boundary conditions, Discrete Contin. Dyn. Syst. Ser. S 6, no. 5 (2013), 1259-1275 (2013).

[17] Y. GigA, Solutions for semilinear parabolic equations in $L^{p}$ and regularity of weak solutions of the Navier-Stokes system, J. Differential Equations, 62 (1986), 186-212.

[18] Y. Giga, T. MiYaKawa Solutions in $L_{r}$ of the Navier-Stokes initial value problem, Arch. Rational Mech. Anal., 89, (1985), 267-281.

[19] Y. Giga, H. SoHR, On the Stokes operator in exterior domains, J. Fac. Sci. Univ. Tokyo Sect. IA Math. 36, no. 1 (1989) 103-130.

[20] Y. Giga, H. Sohr, Abstract $L^{p}$-estimates for the Cauchy Problem with applications to the NavierStokes equations in Exterior Domains, J. Funct. Anal., 102, no. 1 (1991) 72-94.

[21] T. KATO, Strong $L^{p}$ solutions of the Navier-Stokes equation in $R^{m}$, with applications to weak solutions, Math. Z. 187 (1984), 471-480.

[22] P. C. Kunstmann L. Weis. Maximal $L_{p}$ regularity for Parabolic Equations, Fourier Multiplier Theorems and $H^{\infty}$ functional Calculus, Functional Analytic Methods for Evolution Equations, Lecture Notes in Mathematics, 1855, Springer Berlin Heidelberg (2004) 65-311, doi:10.1007/978-3-54044653-8-2.

[23] J. LERAY, Sur le mouvement d'un liquide visqueux emplissant l'espace, Acta Math. 63 (1934), 193248.

[24] M. Mitrea, S. Monniaux, On the analyticity of the semigroup generated by the Stokes operator with Neumann-type boundary conditions on Lipschitz subdomains of Riemannian manifolds, Trans. Amer. Math. Soc. Volume 361, 3125-3157, 2009.

[25] M. Mitrea, S. Monniaux, M. Wright, The Stokes operator with Neumann boundary conditions in Lipschitz domains, J. Math. Sci. (N.Y.) Volume 176, 409-457, 2011.

[26] S. Monniaux, E. M. Ouhabaz, The incompressible Navier-Stokes system with time-dependent Robin-type boundary conditions, J. Math. Fluid Mech., Volume 17, 707-722, 2015.

[27] C.L.M.H. NAVIER, Mémoire sur les lois du mouvement des fluides, Mem. Acad. R. Sci. Inst. 6, 389416 (1823).

[28] J. Neustupa, P. Penel, The Navier-Stokes equation with inhomogeneous boundary conditions based on vorticity, Parabolic and Navier-Stokes equations. Part 2, Banach Center Publ. Volume 81, Polish Acad. Sci. Inst. Math., Warsaw, 321-335, 2008.

[29] A. PAZY, Semi-groups of linear operators and applications to partial differential equations, SpringerVerlag, New-York, Inc. (1983).

[30] J. SAAL, Stokes and Navier Stokes equations with Robin boundary conditions in a half space, J. Math. Fluid Mech. 8, no. 2 (2006) 211-241.

[31] R. SHIMADA, On the $L^{p}-L^{q}$ maximal regularity for the Stokes equations Robin boundary condition in a bounded domain, Math. Methods Appl. Sci. 30, no. 3 (2007), 257-289.

[32] C. G. Simader, H. SoHr, A new approach to the Helmholtz decomposition and the Neumann Problem in $L^{q}$-spaces for bounded and exterior domains, Adv. Math. Appl. Sci. 11 (1992) 1-35.

[33] V. A. SolonNiKov, Estimates for solutions of nonsatationary Navier-Stokes equation, J. Sov. Math. 8 (1977), 467-529. 